\title{
Os impactos da modularidade nos Sistemas de Inovação: uma análise da literatura
}

The impacts of modularity in Innovation Systems: a literature review

\author{
Suzana Regina Moro \\ suzana.moro19@gmail.com \\ Universidade Federal de Santa Catarina (UFSC) \\ Mauricio Uriona Maldonado \\ m.uriona@ufsc.br \\ Universidade Federal de Santa Catarina (UFSC)
}

\begin{abstract}
Resumo: A modularidade vem sendo muito utilizada para o desenvolvimento de produtos e serviços, devido aos benefícios percebidos pelas organizações, já que favorece principalmente a integração para o desenvolvimento dentro de redes de colaboração. O objetivo deste artigo é identificar, com base em uma análise da literatura, os impactos do uso da modularidade nos Sistemas de Inovação. Para tal foi feita uma revisão bibliográfica sistemática visando identificar publicações que abordam a relação entre a adoção de estratégias modulares e os Sistemas de Inovação. Foram selecionados 45 artigos que tratam do assunto, dentro das perspectivas nacional, regional, setorial e tecnológica, sendo assim apresentada a análise referente aos impactos em cada um dos níveis dos Sistemas de Inovação e uma agenda para pesquisas futuras.
\end{abstract}

Palavras-chave: Sistemas de Inovação; Modularidade; Arquitetura Modular; Análise da literatura

Códigos JEL: L15; 030.

\begin{abstract}
Modularity has been widely used for products and services development, due to the benefits perceived by the organizations, since it favors mainly the integration for the development within networks of collaboration. The objective of this article is to identify, based on a literature review, the impacts of the use of modularity in Innovation Systems. For this, a systematic bibliographic review was carried out to identify publications that deal with the relationship between the adoption of modular strategies and Innovation Systems. 45 articles were selected that deal with the subject, within the national, regional, sectoral and technological perspectives, thus presenting the analysis regarding the impacts at each level of the innovation systems and an agenda for future research.
\end{abstract}

Keywords: Innovation Systems; Modularity; Modular Architecture; Literature Analysis

JEL Codes: L15; 030.

Recebido em: 23-10-2017. Aceito em: 16-04-2018.

\section{INTRODUÇÃO}

A arquitetura modular é uma abordagem que facilita a fabricação de subconjuntos de módulos que logo podem mais facilmente ser integrados para a montagem final de produtos. Esta estratégia tem sido apontada como aceleradora do processo de inovação, pois acaba reduzindo a complexidade de fabricação de produtos (ULRICH, 1995), motivo pelo qual cada vez mais organizações adotam a modularidade como estratégia competitiva 
(HARMANCIOGLU, 2009).

Um sistema modular pode facilitar o teste simultâneo de muitas abordagens e pode permitir múltiplas entradas para novas ideias (ZHU et al., 2014). Nesse contexto, a arquitetura modular tem sido considerada uma importante perspectiva na pesquisa de inovação de produtos (SHAMSUZZOHA et al., 2013). A modularidade pode estruturar o compartilhamento e a integração do conhecimento (ZHU et al., 2014), diminuir o tempo de desenvolvimento de novos produtos (SANCHEZ; MAHONEY, 1996; DANESE; FILIPPINI, 2013), e melhorar o desempenho e características do produto (ULRICH, 1995; LAU et al., 2010; DANESE; FILIPPINI, 2013). O uso da arquitetura modular facilita ainda o fornecimento, fabricação e integração na concepção, simplificando a comunicação e o compartilhamento de informação, construindo uma relação de confiança entre os parceiros da cadeia de suprimentos (JACOBS et al., 2007).

A modularidade é importante para a colaboração no projeto porque módulos separados podem ser trabalhados de forma independente e em paralelo, e os projetistas trabalhando em módulos diferentes não têm que estar localizados num mesmo local (BALDWIN; VON HIPPEL, 2011). Cada módulo de conhecimento pode levar a inovação simultaneamente, dentro das regras estabelecidas do sistema (ZHU et al., 2014). Sendo assim, quando os produtos são modulares, menos troca de informações é necessária porque esta informação já está disponível (CABIGIOSU et al., 2015).

Neste artigo o interesse é centrado na visualização da estratégia de modularidade a nível do sistema de inovação, considerando que existem diversas camadas ou dimensões destes sistemas. A pergunta de pesquisa foca em entender como estão sendo utilizadas as estratégias de modularidade nas diferentes camadas do sistema de inovação? Em especial, considerando o fato de existir um interesse crescente, na área de sistemas de inovação, sobre estudos que visem a interdisciplinaridade (GODIN, 2006) bem como da interface com outras abordagens, tais como a das Cadeias Globais de Valor (JUROWETZKI et al., 2018), onde a modularidade pode representar um ganho competitivo e uma forma adicional de extrair valor desses relacionamentos globais.

Nesse sentido, o objetivo deste trabalho é analisar a literatura, identificando os estudos que abordam a relação e impacto da arquitetura modular no Sistema de Inovação. O restante deste trabalho está estruturado da seguinte forma: na próxima seção são apresentados os principais conceitos referentes à modularidade e os níveis de classificação dos Sistemas de 


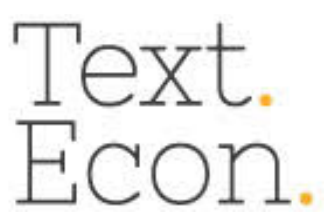

http://dx.doi.org/10.5007/2175-8085.2018v21n2p01

Inovação que foram usados para este estudo. Na seção 3 são descritos os métodos adotados para a revisão da literatura. A seção 4 apresenta a análise referente aos impactos do uso da arquitetura modular em cada um dos níveis dos Sistemas de Inovação. Na seção 5 são discutidos os principais resultados da análise, bem como outras perspectivas para trabalhos futuros. O trabalho finaliza com as conclusões na seção 6 , relativas à adoção da modularidade e os impactos nos Sistemas de Inovação.

\section{CONCEITOS BÁSICOS DE MODULARIDADE E SISTEMAS DE INOVAÇÃO}

Esta seção está dividida em 2 subseções. Inicialmente são apresentados os principais conceitos da arquitetura modular identificados na literatura. Na sequência serão apresentados os conceitos de Sistemas de Inovação em cada um dos níveis: nacional, regional, setorial e tecnológico, que foram utilizados para classificação das publicações e análise de conteúdo.

\subsection{Modularidade e conceitos associados}

A arquitetura modular é uma abordagem que subdivide um sistema em módulos que podem ser criados de forma independente e, serem utilizados em sistemas diferentes para funcionalidades diversas (ULRICH, 1995). A escolha da arquitetura de um produto - maneira pela qual os elementos funcionais de um produto são atribuídos aos componentes físicos e a forma pela qual esses componentes interagem - é importante na tomada de decisão gerencial e pode ser um fator-chave para o desempenho das organizações (ULRICH, 1995; FIXSON, 2005). Essa escolha depende da empresa, do mercado e das características do produto, além da estrutura da cadeia de fornecimento (ÜLKÜ; SCHMIDT, 2011).

Organizações modulares alcançam a decomposição através do uso de pequenas unidades dentro de uma organização maior, sendo que cada unidade é responsável por um domínio específico, de maneira quase independente e tomando decisões autônomas sobre várias questões dos negócios (BENASSI, 2009). Geralmente assume-se que produtos e organizações modulares estão relacionados (LANGLOIS, 2002; HOETKER, 2006). No geral, os produtos modulares devem ser concebidos e produzidos por organizações modulares, enquanto os produtos integrais devem ser produzidos por organizações integrais caracterizadas por relações estreitas e compartilhamento de conhecimento (SANCHEZ; 
MAHONEY, 1996). Organizações modulares lidam com fornecedores diretamente, permitindo maior eficácia e flexibilidade através do agrupamento de recursos, atribuição de domínio específico, e a autonomia nos processos de tomada de decisão (BENASSI, 2009). O fornecimento modular envolve complexidade, necessidade de concentração em atividades essenciais e capacidade de reconhecer e organizar atividades que não são consideradas essenciais para o fornecimento dos módulos (DORAN et al., 2007).

A rede de inovação modular é um sistema de sinergia orgânica de acordo com as regras da modularidade (BALDWIN; CLARK, 1997). Com base na rede de inovação modular, o sistema de gestão do conhecimento pode coordenar a aprendizagem organizacional flexível nas empresas e integração de inovação aberta além das fronteiras organizacionais (QI et al., 2012). A rede de inovação modular confere vários benefícios como a redução dos custos de transação de conhecimento sobre produtos, redução no custo de busca de conhecimento complementar, encurtando o tempo de desenvolvimento e reduzindo a complexidade dos sistemas de conhecimento (QI et al., 2012).

\subsection{Sistemas de Inovação e sua classificação em níveis}

Para esse estudo considerou-se o conceito de Inovação sistêmica, de origem Neoschumpeteriana. Nesse contexto, os Sistemas de Inovação são uma rede de instituições nos setores público e privado (FREEMAN, 1995), cuja principal função é desenvolver, difundir e utilizar as inovações (EDQUIST, 2004). Pode-se claramente diferenciar os Sistemas de Inovação em quatro níveis: nacional, regional, setorial e tecnológico (HEKKERT et al., 2007; NIOSI, 2010), que foram utilizados como base neste estudo.

Um Sistema Nacional de Inovação (NIS) considera os limites geográficos fixos dos estados/ nações como o limite natural do sistema de inovação (LUNDVALL et al., 2002; EDQUIST, 2004). Usando as fronteiras nacionais, são identificados os atores que partilham aspectos comuns como cultura, história, língua, instituições sociais e políticas (EDQUIST, 2004). Assim, o foco do NIS é identificar a importância das interações entre os agentes dentro de um único país e a maneira que eles apoiam a aprendizagem e promovem a inovação (NIOSI, 2010).

A abordagem de Sistema Regional de Inovação (RIS) foi desenvolvida, sendo a ideia básica semelhante à da abordagem do NIS, utilizando como unidade de análise uma região 
(COOKE et al., 1997). O RIS é um conjunto de instituições (empresas inovadoras, universidades, fundações de pesquisa, agências de fomento à pesquisa, laboratórios governamentais e outros órgãos pertinentes do governo) e o fluxo de conhecimento, pessoal, verbas de pesquisa, regulação e incorporação de tecnologia que ocorrem dentro de uma região (NIOSI, 2010). A principal contribuição de estudiosos sobre RIS é a observação de que a distância geográfica entre os atores tem um efeito significativo no desempenho inovador da região (HEKKERT; NEGRO, 2011). Com o tempo, a literatura sobre os RIS fundiu-se com a sobre economia regional e geográfica, clusters, regiões de aprendizagem, distritos industriais e outros conceitos, possibilitando construir hipóteses e compartilhar informações (NIOSI, 2010). As abordagens de NIS e RIS, normalmente, não consideram uma análise detalhada dos processos de inovação tecnológica (HEKKERT; NEGRO, 2011). A política regional de inovação exige um foco nos sistemas regionais e compreensão da evolução dos sistemas tecnológicos globais ou sistemas setoriais é fundamental quando se trata de definir as necessidades da coordenação supranacional e definição de regras (LUNDVALL et al., 2002).

O conceito de Sistema Setorial de Inovação (SIS) ganhou destaque no fim da década de 1990 e se espalhou rapidamente (MALERBA, 2002). Seu principal pressuposto subjacente é que a inovação acontece não só a nível regional ou nacional, mas também a nível global (NIOSI, 2010). O foco do SIS é nos agentes e organizações, colocando ênfase nas interações não comerciais e nos processos de transformação do sistema (MALERBA, 2002). Mais precisamente, um SIS pode ser definido como um sistema de organizações ativas no desenvolvimento e fabricação de produtos de um setor e na geração e utilização de uma tecnologia do setor (MALERBA, 2002; MALERBA, 2004). A principal preocupação reside na dinâmica global das empresas ativas de um setor e os limites para o SIS emergem das condições específicas de cada setor, centrando-se sobre as fontes de conhecimento e sobre o papel desempenhado pelo espaço geográfico nos processos de transmissão do conhecimento (MALERBA, 2004).

Contudo, a dinâmica de um SIS, incluindo os seus limites espaciais, deriva do seu regime tecnológico, que consiste nas condições de oportunidade e apropriabilidade, a natureza e a forma de acumulação do conhecimento técnico, e os meios de transmissão e comunicação do conhecimento (NIOSI, 2010). No caso de se estudar a dinâmica das inovações, com o principal interesse de entender e analisar uma evolução tecnológica específica, o sistema de inovação adequado é o Sistema de Inovação Tecnológico (TIS) (HEKKERT; NEGRO, 2011). 


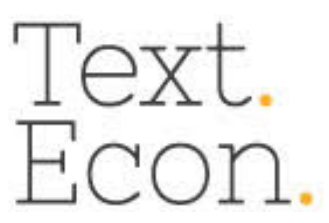

http://dx.doi.org/10.5007/2175-8085.2018v21n2p01

Este conceito permite estudar as características do sistema associado com uma tecnologia emergente específica, para analisar seus pontos fortes e fracos, bem como sua dinâmica, e compará-lo com um sistema de tecnologia incumbente (JACOBSSON; JOHNSON, 2000).

\section{MÉTODOS ADOTADOS}

A revisão sistemática da literatura é um meio de identificar, avaliar e interpretar as pesquisas disponíveis relevantes para uma questão de pesquisa específica, ou área de assunto ou fenômeno de interesse (KITCHENHAM, 2004). O foco desta revisão foi a análise de conteúdo, que é utilizada para a descrição sistemática do conteúdo da literatura em um campo específico ou sobre um determinado assunto (SEURING et al., 2005).

Inicialmente foram feitos testes para busca com as palavras-chaves "modular*" e "system* innovation". Nessa busca foram encontrados respectivamente 4 e 6 artigos. Excluindo-se os artigos duplicados restaram apenas 7 artigos. Esses artigos abordam em geral o uso de ferramentas específicas ou apresentam estudos de caso focado em apenas um tipo de sistema de inovação, não possibilitando traçar conclusões gerais a respeito do assunto, sendo necessária uma busca mais ampla. Dessa maneira, posteriormente, a revisão da literatura foi conduzida conforme apresenta a Figura 1.

Figura 1 - Procedimentos utilizados para a revisão de literatura 
1- Definição dos procedimentos para a coleta de dados

- Bases de dados: ISI Web of Science da Thomson Reuters e Scopus da Elsevier

- Artigos ou revisões

- Sem delimitação de data

- Palavras-chave foram buscadas no título, palavras -chave e resumo: innovation" e palavras-chave rela cionadascom modularidade: " component modularity"; "component innovation"; "modular decomposition"; "modular production"; "modular design"; "product architecture "; "product modularity"; "modular construction"; " modular architecture"; "decomposability"; " modular approach"; "modular structure"; "modular model"; "modular-in-use"; "modular product structure "; "modular system"; "mass customization"; "design and customization"; " modular manufacturing"; " near decomposity"; "technology modularization" e "module* and design"

2- Seleção dos artigos coletados

- Exclusão dos duplicados= 2456 a rtigos

- Leitura do título do artigo e palavras -cha ve $=1190$ a rtigos

- Leitura do resumo= 388

- Leitura na íntegra= 67

\section{3- Identificação de tópicos relevantes,}

análise e interpretação dos resultados

- Foram definidas as classificações para os a rtigos de a cordo com os níveis dos sistemas de inovação

- Final=45 a rtigos utilizados

Fonte: elaborado pelos autores.

Os artigos selecionados, que abordam a relação da modularidade de acordo com os Sistemas de Inovação foram agrupados conforme os níveis: nacional, regional, setorial e tecnológico. Na sequência são apresentados os resultados referentes a cada nível dos Sistemas de Inovação e as perspectivas de pesquisas futuras identificadas na literatura.

\section{ANÁLISE DE CONTEÚDO}

O Quadro 1 apresenta as publicações selecionadas no estudo e os respectivos níveis abordados por cada publicação: nacional, regional, setorial e tecnológico.

Quadro 1 - Publicações selecionadas para o estudo e níveis dos Sistemas de Inovação abordados

\begin{tabular}{|c|c|c|c|c|}
\hline Referências & NIS & RIS & SIS & TIS \\
\hline Baldwin e Henkel (2015) & $\mathrm{X}$ & & & $\mathrm{X}$ \\
\hline Bouncken et al. $(2015)$ & & & & $\mathrm{X}$ \\
\hline Cabigiosu et al. (2015) & & & $\mathrm{X}$ & \\
\hline Liu et al. (2015) & $\mathrm{X}$ & $\mathrm{X}$ & $\mathrm{X}$ & $\mathrm{X}$ \\
\hline Meng (2015) & & & $\mathrm{X}$ & \\
\hline Sun e Gong (2015) & $\mathrm{X}$ & $\mathrm{X}$ & & \\
\hline Bergek et al. $(2014)$ & $\mathrm{X}$ & & & \\
\hline Zeschky et al. $(2014)$ & & $\mathrm{X}$ & $\mathrm{X}$ & \\
\hline
\end{tabular}




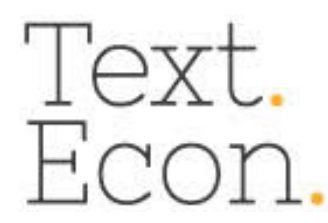

http://dx.doi.org/10.5007/2175-8085.2018v21n2p01

\begin{tabular}{|c|c|c|c|c|}
\hline Zhu et al. (2014) & & & $\mathrm{X}$ & $\mathrm{X}$ \\
\hline Cabigiosu et al. (2013) & & & & $\mathrm{X}$ \\
\hline Danese e Filippini (2013) & & & $\mathrm{X}$ & \\
\hline Davies e Joglekar (2013) & & & $\mathrm{X}$ & \\
\hline Ethiraj e Posen (2013) & & & & $\mathrm{X}$ \\
\hline Henkel et al. (2013) & & & & $\mathrm{X}$ \\
\hline Kamrad et al. (2013) & & & & $\mathrm{X}$ \\
\hline Mcdermott et al. (2013) & & & $\mathrm{X}$ & \\
\hline Mlecnik (2013) & & & $\mathrm{X}$ & \\
\hline Ravishankar e Pan (2013) & & & $\mathrm{X}$ & \\
\hline Salvador e Villena (2013) & & & & $\mathrm{X}$ \\
\hline Song et al. (2013) & & & $\mathrm{X}$ & $\mathrm{X}$ \\
\hline Cabigiosu e Camuffo (2012) & & & $\mathrm{X}$ & \\
\hline Maccormack et al. (2012) & & & $\mathrm{X}$ & \\
\hline Rotaba e Beaudry (2012) & $\mathrm{X}$ & & & \\
\hline Cheng (2011) & & & $\mathrm{X}$ & \\
\hline Lau et al. (2011) & & & $\mathrm{X}$ & \\
\hline Ozman (2011) & & & $\mathrm{X}$ & \\
\hline Ülkü e Schmidt (2011) & & & $\mathrm{X}$ & \\
\hline Zirpoli e Becker (2011) & & & $\mathrm{X}$ & \\
\hline Danese e Filippini (2010) & & & $\mathrm{X}$ & \\
\hline Campagnolo e Camuffo (2010) & & & $\mathrm{X}$ & \\
\hline Lau et al. $(2010)$ & & & $\mathrm{X}$ & \\
\hline Zhang e Gao (2010) & & & $\mathrm{X}$ & \\
\hline Benassi (2009) & & & $\mathrm{X}$ & \\
\hline Doran e Hill (2009) & & & $\mathrm{X}$ & \\
\hline Frigant e Layan (2009) & & $\mathrm{X}$ & $\mathrm{X}$ & \\
\hline Harmancioglu (2009) & $\mathrm{X}$ & & & \\
\hline Takeda et al. (2008) & & $\mathrm{X}$ & & \\
\hline Doran et al. (2007) & & & $\mathrm{X}$ & \\
\hline Vind e Fold (2007) & $\mathrm{X}$ & & & \\
\hline Andersen (2006) & $\mathrm{X}$ & $\mathrm{X}$ & & \\
\hline Mikkola (2003) & & & $\mathrm{X}$ & \\
\hline Sturgeon $(2002)$ & $\mathrm{X}$ & & & \\
\hline Schilling e Steensma (2001) & & & & $\mathrm{X}$ \\
\hline Baldwin e Clark (1997) & & & & $\mathrm{X}$ \\
\hline Sanchez e Mahoney (1996) & & & $\mathrm{X}$ & $\mathrm{X}$ \\
\hline
\end{tabular}

Fonte: elaborado pelos autores com base na análise da literatura.

\subsection{Modularidade no Sistema Nacional de Inovação (NIS)}

Motivados pela onda de novas economias industrializadas, a liberalização do comércio, e diminuição dos custos de transporte e comunicação, as atividades de produção mudaram dos países de altos salários para os países de baixos salários (ANDERSEN, 2006). Indústrias de alta tecnologia nas principais economias dependem da modularidade do produto para terceirizar vários componentes, provavelmente, em empresas de economias emergentes (ROTABA; BEAUDRY, 2012). As economias emergentes enfatizam a utilização de parcerias 
com universidades para produzir conhecimento (ROTABA; BEAUDRY, 2012). Dessa forma, torna-se importante que os governos de economias emergentes considerem a formulação de políticas industriais para fomentar a adoção da modularização (LIU et al., 2015). É importante ainda estimular a difusão dos benefícios da adoção da arquitetura modular junto a universidades, que por sua vez tornar-se-ão difusoras do conhecimento.

Como forma de promover o desenvolvimento econômico, a formulação de políticas industriais pode priorizar principalmente as empresas de menor porte. As micro e pequenas empresas devem fazer pleno uso dos recursos de informação, das vantagens de flexibilidade, participar ativamente de redes de inovação e buscar oportunidades de desenvolvimento dentro das oportunidades identificadas no sistema modular (SUN; GONG, 2015). Já as empresas de pequeno e médio porte devem tirar proveito de suas habilidades únicas e vantagens profissionais, desenvolvendo ativamente associações, para se tornar empresas de plataforma modular, e ter acesso ao domínio do negócio (SUN; GONG, 2015). Bergek et al. (2014) relatam que a utilização de instrumentos políticos gerais regulamentadores gerou melhorias com base na inovação modular em empresas do setor de energia e automobilístico.

As organizações estão cada vez mais mudando para o fornecimento global de seus componentes e/ou projetos em vez de gastar milhões de dólares para projetar e desenvolvê-los internamente (HARMANCIOGLU, 2009). Vind e Fold (2007) com base na rede global de produção na indústria de eletrônicos dos EUA construíram um modelo de organização para a rede de Singapura, com base na produção modular, e argumentam que a manutenção e desenvolvimento futuro de redes produtivas nacionais juntamente com políticas industriais do Estado são capazes de corresponder aos requisitos determinados pela dinâmica global (VIND; FOLD, 2007). Sturgeon (2002) define a indústria eletrônica os EUA como tendo o mais alto nível de modularidade devido ao elevado grau de abertura e dispersão geográfica.

Quando as leis de propriedade intelectual no país são ineficazes ou inacessíveis financeiramente, a modularidade pode ser usada para ocultar informações e, assim, proteger a propriedade intelectual (BALDWIN; HENKEL, 2015). Nesse contexto, a modularidade acaba funcionando como um mecanismo de resposta à ineficiência das políticas de proteção da propriedade industrial, contudo se percebida pelo Estado, podem ser direcionados esforços para oportunizar tal uso. 


\section{Text.}

http://dx.doi.org/10.5007/2175-8085.2018v21n2p01

\subsection{Modularidade no Sistema Regional de Inovação (RIS)}

Em um SRI, uma rede inter-organizacional densa é reconhecida como um fator chave na promoção da difusão do conhecimento, aprendizagem regional e transferência de recursos eficazes (TAKEDA et al., 2008). Inspirados nos estudos de clusters regionais de alta tecnologia dos Estados Unidos, muitos países enfatizam a criação de aglomerados regionais de empresas inovadoras em torno de universidades, a fim de estimular o desenvolvimento econômico regional (ANDERSEN, 2006). A modularidade permite que empresas locais quebrem a barreira tecnológica em indústrias com uso intensivo de tecnologia (LIU et al., 2015). Em organizações de Pesquisa e Desenvolvimento (P\&D) global, quanto a maior utilização de modularização do produto, maior também será a habilidade da organização para a adaptação dos produtos para os requisitos do mercado local (ZESCHKY et al., 2014).

A geografia das organizações decorre de sua exploração das vantagens locais e de interação das restrições de proximidade, que deve ocorrer através de uma análise afinada e completa (FRIGANT; LAYAN, 2009). Os clusters regionais podem desfrutar de vantagens competitivas, já que há uma tendência para as empresas transacionarem com aqueles em estreita proximidade, portanto compreender a estrutura da rede e a proximidade física das organizações é essencial (TAKEDA et al., 2008). Tanto os decisores políticos quanto os gestores devem desafiar velhas suposições sobre a dinâmica da inovação em clusters regionais e abraçar uma visão mais multifacetada e dinâmica, a fim de oferecer novas e úteis perspectivas na transferência global (ANDERSEN, 2006).

A atração de empresas líderes para uma região com infraestrutura abundante, desenvolve a economia regional, porque elas constroem uma grande rede modular de fornecedores e clientes na região, trazendo uma ampla gama de efeitos positivos para o restante da economia regional, através das interações com outras empresas na rede, difusão de conhecimento, aprendizagem focalizada, transferência de recursos, ações coletivas e transações comerciais (TAKEDA et al., 2008). Dessa forma, os formuladores de políticas do governo devem se concentrar em corresponder às empresas, ajudando-as a construir uma rede de inovação modular, promovendo os clusters industriais e o desenvolvimento dos RIS (SUN; GONG, 2015).

\subsection{Modularidade no Sistema Setorial de Inovação (SIS)}




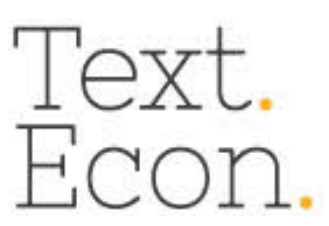

http://dx.doi.org/10.5007/2175-8085.2018v21n2p01

A modularidade permite a coordenação através da adesão a objetivos partilhados e normas comuns, conectando desenvolvedores geograficamente dispersos (MIKKOLA, 2003). A produção modular oferece uma chance de modificar geograficamente a cadeia de valor (FRIGANT; LAYAN, 2009). Além disso, em uma cadeia de fornecimento descentralizada, a natureza da relação entre o fabricante de equipamento original e seu fornecedor (concorrente ou colaborativo) desempenha um papel na escolha da arquitetura do produto: arquiteturas modulares são mais prováveis quando as partes têm relações de concorrência, enquanto relações de longo prazo e com maior confiança facilitam arquiteturas produtos mais integrais (ÜLKÜ; SCHMIDT, 2011).

A abordagem de cadeias de valor global (Global Value Chain- GVC) aborda as circunstâncias externas (globais) do desenvolvimento econômico nos países. Os estudos sobre GVC geralmente são delineados por fronteiras setoriais e em setores de baixa tecnologia (JUROWETZKI et al., 2018). Nesse sentido, os estudos sobre GVC estão inseridos no contexto setorial dos Sistemas de Inovação. O SIS está relacionado de duas maneiras: através de processos de interação e cooperação no desenvolvimento de artefatos tecnológicos e através de processos de concorrência e seleção na inovação e atividades de mercado (MALERBA, 2002; 2004), que serão tratados na sequência.

\subsubsection{Integração e cooperação no Sistema Setorial de Inovação}

Para introduzir com sucesso inovações, os fornecedores precisam unir forças com outras organizações e responder aos desafios da inovação sistêmica (MLECNIK, 2013). A fim de gerir a complexidade associada à criação e funcionamento de redes internacionais, as organizações devem delegar a responsabilidade de atividades significativas para agentes externos, e podem conseguir isto através da concepção ou reengenharia de seus produtos ou serviços como subconjuntos modulares que podem ser divididos com uma rede de organizações parceiras (MCDERMOTT et al., 2013). Quando a empresa desenvolve produtos modulares, requer maior envolvimento tanto dos fornecedores quanto dos clientes, assim, se uma empresa quer desenvolver produtos modulares, deve integrar canais de coordenação adicionais em toda a cadeia de fornecimento (LAU, 2011). O controle rígido e o intenso monitoramento do comportamento do fornecedor se torna menos necessário, já que a interdependência é reduzida (SANCHEZ; MAHONEY, 1996). 
A modularidade do produto quer em conjunto ou independente da integração pode produzir flexibilidade para as empresas dentro de uma cadeia de fornecimento (DAVIES; JOGLEKAR, 2013). Doran et al. (2007) examinou o desenvolvimento em uma cadeia de fornecimento modular e observou uma série de características distintas associadas com a estratégia modular. A adoção de uma estratégia modular tem implicações para todos os envolvidos na cadeia de suprimentos (DORAN; HILL, 2009). Inicialmente, o fornecimento modular envolve complexidade, a necessidade de concentração das atividades no núcleo do módulo, e a necessidade de reorganizar as atividades que não são considerados críticas para o fornecimento de módulos (DORAN et al., 2007).

Realizando uma pesquisa com 251 fabricantes em Hong Kong, Lau (2011) verificou que o projeto modular, a inovação de produtos e coordenação interna são positivamente correlacionados com o envolvimento de fornecedores e de clientes, e esse envolvimento leva ao melhor desempenho dos novos produtos. As relações com fornecedores em sistemas de produtos modulares são uma oportunidade importante para as empresas aprofundarem o seu conhecimento no nível dos componentes, e às vezes no nível do sistema (OZMAN, 2011). A modularidade cria uma série de oportunidades de colaboração mais forte com os fornecedores e aprendizagem entre empresas, facilitando práticas de integração de fornecedores no desenvolvimento de novos produtos, que por sua vez pode levar a melhorias adicionais de desempenho (DANESE; FILIPPINI, 2013).

As vantagens da economia de escopo ajudam as organizações modulares a economizar com o compartilhamento de recursos e conhecimentos da cadeia de suprimentos e aumentar a escala de diversificação (CHENG, 2011). A implicação prática para os gestores é que a redução de do tempo de desenvolvimento de novos produtos requer simultaneamente a modularidade do produto e integração interfuncional (DANESE; FILIPPINI, 2010). A medida que ocorre tal modularização varia de acordo com os direcionadores técnicos e de engenharia e considerações estratégicas, e ainda entre o tipo de processo e produto indústrias, a variedade de saída, tamanho do lote, e o do poder de negociação entre o fornecedor e os compradores na cadeia de valor, variando desta forma de entre tipos de indústrias (MCDERMOTT et al., 2013).

O grau de modularidade dos componentes impacta negativamente o compartilhamento de informação entre o comprador e o fornecedor e o desempenho das relações de fornecimento (CABIGIOSU; CAMUFFO, 2012). Produtos desenvolvidos por empresas 
fracamente unidas são significativamente mais modulares que os desenvolvidos por empresas fortemente unidas em uma rede de inovação (MACCORMACK et al., 2012). Dependendo das estratégias das empresas, estruturas organizacionais e capacidades, ao nível da empresa, maior utilização da modularidade do produto pode estar associada tanto com a menor partilha de informação com os fornecedores como com maior partilha de informação com os fornecedores (CABIGIOSU; CAMUFFO, 2012). Os compradores e fornecedores podem ser fracamente unidos se utilizarem projetos de produtos modulares no que diz respeito a partilha de conhecimento, mas não na partilha de informação (CABIGIOSU et al., 2015).

A modularidade pode ser um dispositivo para construir uma cadeia de suprimentos e tornar a organização rentável (CHENG, 2011). A modularidade nas organizações pode facilitar a criação e o desenvolvimento das capacidades dinâmicas (RAVISHANKAR; PAN, 2013). A integração de conhecimento baseada em ontologias aumenta a eficiência da integração e reutilização de conhecimento entre os diferentes agentes da inovação e evita dispersões desnecessárias de ativos intelectuais (SONG et al., 2013). Paradoxalmente, no entanto, a gestão modular também pode sufocar o potencial estratégico de tais capacidades por entrar em conflito com a integração horizontal das unidades (RAVISHANKAR; PAN, 2013). A modularidade do produto pode gerar um maior envolvimento dos fornecedores no desenvolvimento de novos produtos, que por sua vez pode determinar melhorias no tempo de desenvolvimento e desempenho do produto (DANESE; FILIPPINI, 2013).

Instituir organizações modulares pode aumentar a eficiência na utilização da capacidade, pois os recursos internos podem ser utilizados em sinergia com os dos membros da cadeia de fornecimento (CHENG, 2011). O modo de gestão de convergência da empresa para a modularidade nas cadeias de fornecimento estimula a saída rápida de conhecimentos e experiência de gestão, transmissão rápida de informações e relações de oferta e demanda nas cadeias de fornecimento, as quais são benéficas para a inovação rápida de produtos nas organizações da cadeia de fornecimento e para impulsionar os produtos modulares serem executados rapidamente e economicamente (MENG, 2015). Os benefícios desejáveis destas relações não serão imediatamente percebidos até que os parceiros comerciais atingiram uma integração mais completa (CHENG, 2011).

O projeto modular está relacionado a uma cadeia de fornecimento fracamente coordenada, enquanto projeto integral está associado a uma cadeia de fornecimento bem coordenada (LAU et al., 2010). Empresas que adotam uma organização modular podem 


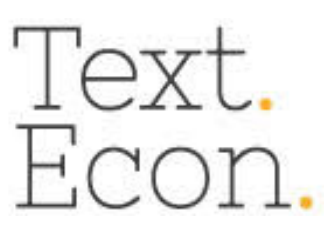

http://dx.doi.org/10.5007/2175-8085.2018v21n2p01

enfrentar o risco de se tornar uma constelação de empresas (mal) relacionadas (BENASSI, 2009). Estudos sobre a modularização do produto, com raras exceções, postulam uma simetria perfeita entre os módulos de produtos e o particionamento de conhecimento entre os agentes econômicos dentro e entre organizações (CAMPAGNOLO; CAMUFFO, 2010). Empresas que adotam uma organização modular podem ter apenas um conhecimento moderado do que está ocorrendo com e entre outras unidades (BENASSI, 2009). Uma cadeia de fornecimento descentralizada pode ser associada com um produto de arquitetura integral quando a colaboração técnica não é excessiva e os fornecedores têm capacidades de desenvolvimento de produtos significativamente superiores (ÜLKÜ; SCHMIDT, 2011).

O projeto modular do produto é eficaz na resolução da tarefa de coordenar fornecedores e como eles desenvolvem componentes e sistemas de forma independente, que precisam ser integrados fisicamente, assim diminuindo os esforços de coordenação com os fornecedores (ZIRPOLI; BECKER, 2011). As organizações devem reduzir as interdependências entre os módulos internos e terceirizados, especialmente em indústrias caracterizadas por ciclos rápidos e curtos, com ampla concorrência (ZHANG; GAO, 2010). Contudo, alianças, parcerias e joint ventures na cadeia de fornecimento, requerem um período de tempo para apresentar resultados de colaboração bem-sucedida (CHENG, 2011). Se o ciclo de vida do produto é longo o suficiente, os benefícios da integração ou cooperação entre empresas se tornará mais significativo (ZHANG; GAO, 2010).

Em organizações globais de $\mathrm{P} \& \mathrm{D}$, quanto maior utilização de projeto modular, menor será o esforço de coordenação formal e informal da sede para integrar as atividades de P\&D da subsidiária (ZESCHKY et al., 2014). As empresas multinacionais estão cada vez mais reconhecendo a importância de utilizar o conhecimento e as capacidades de um ecossistema de inovação mais amplo, além da sua rede corporativa de subsidiárias (MCDERMOTT et al., 2013). No entanto, esta relação é afetada e explicada por quatro fatores de contingência: novo módulo/ desenvolvimento do componente, dispersão de conhecimento tecnológico e criação, tamanho da equipe de projeto e eficiência da cadeia de suprimentos (LAU et al., 2010). Além disso, antes de decidir investir na modularidade do produto, os gestores devem determinar o nível de integração interfuncional, que é na verdade um pré-requisito para o sucesso da implementação da modularidade do produto (DANESE; FILIPPINI, 2010). 


\section{Text.}

http://dx.doi.org/10.5007/2175-8085.2018v21n2p01

\subsubsection{Concorrência no Sistema Setorial de Inovação}

A modularidade torna possível conduzir inovação paralela entre os concorrentes (ZHU et al., 2014). Porém, percebe-se que com o projeto modular, a concorrência entre as empresas tornou-se consecutiva, o que significa que as empresas que fabricam produtos semelhantes estão desenvolvendo as inovações simultaneamente, levando a intensa competição, mas é essa relação de concorrência que serve como uma força motriz para a modernização de produtos da indústria em geral (ZHU et al., 2014).

Empresas com módulos semelhantes podem conduzir inovação independentes ao mesmo tempo, e depois de uma empresa introduzir uma inovação, outras empresas vão inovar em resposta (ZHU et al., 2014). O uso da arquitetura modular pode também favorecer as empresas retardatárias no desenvolvimento de determinados tipos de produtos, que através da acumulação das experiências na operação de marketing e capacidade de inovação de produtos, podem iniciar uma transformação estratégica, e competir com as principais empresas estabelecidas no mercado, em longo prazo, ganhando vantagens competitivas sustentáveis (LIU et al., 2015). Ainda pode ser salientado que na ausência de modularidade, as empresas devem considerar a transformação interna e a formação de alianças estratégicas para estimular a modularização industrial (LIU et al., 2015).

\subsection{Modularidade no Sistema Tecnológico de Inovação (TIS)}

Através da modularização da tecnologia, componentes altamente interativos estão integrados num módulo, de modo que um sistema complexo pode ser dividido em vários módulos relativamente independentes com interações reduzidas e normalizadas e o grau de complexidade do sistema pode ser reduzido substancialmente (SCHILLING; STEENSMA, 2001). Estruturas de produtos modulares aceleraram diretamente os processos de inovação em alianças e promovem a inovação radical, até certo ponto (BOUNCKEN et al., 2015).

\subsubsection{Integração e cooperação no Sistema Tecnológico de Inovação}

A modularidade também envolve menor divulgação do plano de concepção global, e reduz o risco de vazamento de know-how tecnológico porque o conhecimento dos fornecedores é específico do módulo (SANCHEZ, 1996). Nas indústrias onde a tecnologia muda rapidamente, os fornecedores e compradores podem preferir produtos que podem ser 
modularmente atualizados (KAMRAD et al., 2013). As organizações podem focalizar o uso de recursos nos avanços na tecnologia de dado componente através da concepção e produção apenas desse componente (minimizando, assim, os custos de desenvolvimento e de produção), ao invés de projetar e produzir o produto inteiro como seria necessário com um projeto integral (KAMRAD et al., 2013). A modularidade pode reduzir a complexidade das inovações quando a empresa utiliza rede de inovação, mas o uso demasiado da modularidade pode afetar o grau de novidade do produto (SONG et al., 2013).

A modularidade pode restringir a contribuição inovativa dos fornecedores, mas pode aumentar o controle sobre o desempenho de componentes e substituição do fornecedor (CABIGIOSU et al., 2013). Em relação ao custo de fabricação, as vantagens do envolvimento de fornecedores e da capacidade de desenvolvimento modular enfraquecem quando os produtos e processos são altamente inovadores (SALVADOR; VILLENA, 2013). Para Ethiraj e Posen (2013) quando interdependências de componentes são não-trivial e as empresas são especializados em fabricação de componentes, os retornos da inovação modular não são independentes da taxa e direção da mudança na arquitetura do produto. Para as empresas retardatárias no desenvolvimento de certas tecnologias, um telefone celular, por exemplo, seria um produto relativamente complexo para produzir sem a modularização da tecnologia, mas com a modularização do processo de fabricação de telefones celulares, as atividades de P\&D também são modularizadas (LIU et al., 2015).

Assim, a ação estratégica das partes da empresa para alterar a natureza das dependências tecnológicas entre as empresas em um sistema de produto complexo pode servir não só para mitigar os efeitos negativos das mudanças na arquitetura, mas de fato para gerar mudanças destinadas a mudar a arquitetura de uma forma que pode melhorar significativamente o desempenho em inovação da própria empresa (ETHIRAJ; POSEN, 2013). As indústrias com capacidades superiores de desenvolvimento de produtos modulares se beneficiam mais do envolvimento dos fornecedores em seus esforços para o desenvolvimento de novos produtos em relação ao custo de fabricação e desempenho técnico (SALVADOR; VILLENA, 2013). Os fabricantes interessados em melhorar o desempenho do desenvolvimento de novos produtos através do envolvimento fornecedor também devem prestar muita atenção para o desenvolvimento de suas competências internas e às contingências associadas a cada projeto específico (por exemplo, o grau de inovação) e o tipo de indústria (por exemplo, produtos eletrônicos) (SALVADOR; VILLENA, 2013). 
Um debate importante sobre o uso da arquitetura modular reside na análise da propriedade intelectual. Henkel et al. (2013) argumentam que a proteção intelectual de cada módulo tem que ser cuidadosamente planejada e suas fronteiras devem ser definidas em conformidade. Contudo, os autores ressaltam que em algumas circunstâncias, mesmo com uma arquitetura de produto ou processo modular, a proteção intelectual modular pode não ser necessária, por exemplo, se a estratégia da empresa para se apropriar de valor repousa pesadamente sobre a propriedade de ativos complementares. Da mesma forma, a confiança entre as organizações reduz o risco de comportamento oportunista, e, portanto, pode reduzir a necessidade de prosseguir a propriedade intelectual modular (HENKEL et al., 2013).

\subsubsection{Concorrência no Sistema Tecnológico de Inovação}

Com a inovação paralela devido ao sistema modular, a concorrência entre as empresas torna-se mais intensa, levando as empresas a acelerar o processo de inovação para ficar à frente dos concorrentes (ZHU et al., 2014). Em um sistema modular, as inovações podem ser mais facilmente introduzidas por outras empresas além da criadora da arquitetura modular empresas em mercados relacionados, startups ou mesmo usuários (BALDWIN; CLARK, 1997). No entanto, isto cria uma tensão implícita entre a criação e a apropriação de valor. Ao conceder acesso ao conhecimento de produtos ou processos a colaboradores externos, o criador do sistema modular corre o risco de perder a maior parte do valor para os inovadores complementares (HENKEL et al., 2013). Contratos relacionais tornam possível proteger o conhecimento e manter um monopólio quando os agentes são relativamente pouco confiáveis (BALDWIN; HENKEL, 2015).

Quando as tecnologias de produtos são muito complexas, as empresas tecnologicamente retardatárias podem usar as oportunidades oferecidas pela modularidade e, assim, tentar uma evolução colaborativa da rede de inovação e criar novos modelos de negócios para superar os obstáculos à inovação (LIU et al., 2015). Em seguida, eles podem avançar com inovações disruptivas para atender as demandas de nichos de mercado específicos negligenciadas por empresas líderes já estabelecidas (LIU et al., 2015). Contudo, uma vez que uma estrutura modular é aceita por todas as empresas, não é fácil criar uma grande ruptura, que pode vir a ser um obstáculo à inovação radical (ZHU et al., 2014). 


\section{Text.}

http://dx.doi.org/10.5007/2175-8085.2018v21n2p01

\subsection{Relação dos distintos Sistemas de Inovação e modularidade}

Percebe-se que os estudos sobre os Sistemas de Inovação selecionados em sua maioria analisam os SIS (62\%) e TIS (28\%). Nestes estudos o foco é predominantemente na indústria automotiva. A maioria dos estudos identificados, que foram analisados nos níveis setorial e tecnológico abordam as relações de desenvolvimento e fornecimento entre os envolvidos na produção dos módulos dos produtos, sem salientar o envolvimento de demais agentes, como universidades e outras instituições. Ainda são escassos os estudos empíricos da relação entre o projeto modular e a integração da cadeia de fornecimento (LAU et al., 2010).

Poucos estudos analisaram a modularidade nos níveis nacional $(20 \%)$ e regional (13\%). Considerando o carater geográfico, dizem respeito principalmente a pesquisas realizadas em países desenvolvidos. Assim, considerando a nova Economia Institucional, percebe-se que a maioria se refere a setores industriais presentes em países desenvolvidos. Portanto, estudos em países em desenvolvimento e que se referem a indústrias tipicamente presentes nesses países são escassas.

Ainda é importante salientar que com relação à formulação de políticas, uma área que claramente deve ser revista são os direitos de propriedade intelectual (BALDWIN; VON HIPPEL, 2011). É importante notar que ainda existem algumas limitações no uso do projeto modular, especificamente sua arquitetura rígida e dificuldade para interface, que após o uso e estabelecimento de certos padrões, torna mais difícil para as empresas inovarem radicalmente. A modularidade tendo sido amplamente discutida nos últimos anos, ainda assim, poucos estudos interligam esta com outras áreas do conhecimento. Apesar da ampla ligação entre o projeto modular e os Sistemas de Inovação, poucos estudos foram feitos com esse objetivo de análise específico. Ressaltando assim a importância de maior integração de áreas distintas do conhecimento e de artigos de revisão teórica sobre os temas de maneira conjunta, visando identificar lacunas que possa sem melhor exploradas. Considerando as publicações analisadas, como importante contribuição que a análise de literatura permite propiciar, na sequência são apontadas as principais oportunidades de pesquisa identificadas na literatura analisada.

\section{PERSPECTIVAS DE PESQUISAS FUTURAS}

No que diz respeito aos níveis nacional e regional dos Sistemas de Inovação, 


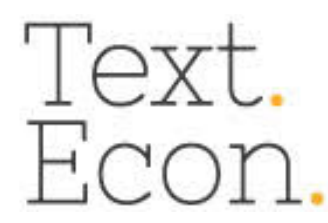

http://dx.doi.org/10.5007/2175-8085.2018v21n2p01

representam lacunas importantes os estudos empíricos de setores e regiões específicos, além de investigações sobre as diferenças entre países no que diz respeito à adoção da arquitetura modular e como esta pode favorecer o desenvolvimento da economia local. Dada a emergência de pesquisas sobre modularidade, é interessante notar que poucas pesquisas tenham objetivamente analisado o desempenho dessa forma ágil da cadeia de fornecimento (CHENG, 2011). Também pode apresentar contribuições interessantes para os estudos de modularidade no âmbito de SIS, integrar com o conceito de GVC. Novos trabalhos empíricos podem tratar o conceito de "rede de produção modular" (CAMPAGNOLO; CAMUFFO, 2010). Provavelmente devido às restrições para coleta de dados e dificuldade de mensuração dos resultados, nenhum estudo analisado avaliou objetivamente os impactos do uso da arquitetura modular na concorrência em sistemas setoriais e tecnológicos. Nesse sentido, os estudos analisados identificaram algumas sugestões de trabalhos futuros, que são apresentadas no Quadro 2.

Quadro 2 - Sugestões de trabalhos futuros identificados

\begin{tabular}{|c|c|}
\hline Referências & Oportunidades de trabalhos futuros \\
\hline Bouncken et al. (2015) & $\begin{array}{l}\text { Analisar outros fatores que podem influenciar o desempenho do uso de produtos } \\
\text { modulares, tais como a gestão de alianças, confiança ou a complexidade do } \\
\text { contrato. }\end{array}$ \\
\hline Cabigiosu et al. (2015) & $\begin{array}{l}\text { Investigar outros setores KIBS, um maior número de empresas e comparar com o } \\
\text { desempenho das empresas. } \\
\text { Validar quantitativamente o estudo realizado. } \\
\text { Utilizar uma abordagem de contingência para explorar como diferentes } \\
\text { caracteristicas das indústrias (por exemplo, o dinamismo tecnológico ou a } \\
\text { disponibilidade de padrões da indústria) pode influenciar a dinâmica entre } \\
\text { comprador e fornecedor, especialmente em termos de partilha de conhecimentos. }\end{array}$ \\
\hline Liu et al. (2015) & $\begin{array}{l}\text { Estudar casos em profundidade com base no quadro analítico sugerido na } \\
\text { pesquisa, e os esforços em comparação transcultural com modelos e estratégias } \\
\text { emergentes em outras economias em desenvolvimento. } \\
\text { Considerar os regimes tecnológicos (isto é, oportunidades tecnológicas, } \\
\text { apropriação da inovação, acúmulo de avanços técnicos e de propriedade } \\
\text { intelectual) e modularidade, bem como efeitos de aprendizagem organizacional } \\
\text { em redes de empresas como antecedentes da inovação disruptiva. } \\
\text { Metodologias para a exploração empírica e teste destes e suas interações precisam } \\
\text { ser desenvolvidos. }\end{array}$ \\
\hline Sun e Gong (2015) & $\begin{array}{l}\text { Utilizar dados de parceria entre as empresas na indústria de serviços de alta } \\
\text { tecnologia, e realizar uma análise quantitativa das falhas estruturais na rede, e } \\
\text { revelar as características de evolução da rede de inovação de serviços de alta } \\
\text { tecnologia em diferentes áreas, e a geração de fomas de condução específicas para } \\
\text { cada tipo de plataforma de empresas. }\end{array}$ \\
\hline Zeschky et al. (2014) & $\begin{array}{l}\text { Realizar estudos envolvendo uma amostra grande de empresas, incluindo outras } \\
\text { indústrias como a farmacêutica, indústria química ou bens de consumo para } \\
\text { confirmar ou avaliar os resultados. } \\
\text { Utilizar outros resultados quantitativos que medem os custos de coordenação (por } \\
\text { exemplo, tempo de trabalho, custos de interação, despesas de viagem, os custos } \\
\text { de logística) pode ajudar as empresas a encontrar uma configuração ideal para a } \\
\text { interação de P\&D globais. }\end{array}$ \\
\hline
\end{tabular}




\begin{tabular}{|c|l|}
\hline & $\begin{array}{l}\text { Investigar potenciais trade-offs entre um projeto refinado de arquitetura de } \\
\text { produtos modulares e os benefícios que estes geram em fases posteriores do } \\
\text { processo de desenvolvimento, particularmente em organizações globais de P\&D. }\end{array}$ \\
\hline Cabigiosu et al. (2013) & $\begin{array}{l}\text { Analisar as configurações da indústria e as especificidades da empresa que } \\
\text { influenciam o papel de modularidade na integração de fontes externas de } \\
\text { inovação. }\end{array}$ \\
\hline \multirow{3}{*}{ Danese e Filippini (2013) } & $\begin{array}{l}\text { Pesquisas empíricas para investigar o impacto da modularidade em inovação de } \\
\text { produtos e inovação dos componentes arquitetônicos em diferentes contextos e } \\
\text { tipos de indústrias. Além disto, o impacto no desempenho do produto em relação } \\
\text { à qualidade e redução de custos. }\end{array}$ \\
\hline Davies e Joglekar (2013) & $\begin{array}{l}\text { Avaliar o impacto da escassez ou excesso de fornecedores, e examinar o } \\
\text { significado da posição da cadeia de abastecimento sobre o desempenho da } \\
\text { empresa que utiliza a modularidade. }\end{array}$ \\
\hline Salvador e Villena (2013) & $\begin{array}{l}\text { Analisar outros efeitos do envolvimento de fornecedores e da capacidade de } \\
\text { desenvolvimento modular no desempenho dos novos produtos, tais como tempo } \\
\text { de mercado e os resultados financeiros de um determinado projeto, bem como } \\
\text { com medidas mais refinadas de inovação de produtos e processos. }\end{array}$ \\
\hline Song et al. (2013) & $\begin{array}{l}\text { Aplicar o modelo proposto de redes de colaboração para a inovação de produtos, } \\
\text { para que tenha maior validade externa, e considerar os problemas em diferentes } \\
\text { fases da inovação de produtos. }\end{array}$ \\
\hline
\end{tabular}

Fonte: elaborado pelos autores com base na análise da literatura.

Conforme apontado pela literatura consultada, existem ainda oportunidades para pesquisa em assuntos relacionados a modularidade e os sistemas de inovação. Poucos estudos empíricos foram realizados até então, que são sugeridos, para que as conclusões possam ser generalizadas (LAU et al., 2010).

\section{CONCLUSÕES}

A natureza complexa e implícita do conhecimento torna a inovação difícil para as organizações, principalmente as estabelecidas mais recentemente, de menor porte e em economias emergentes. A modularização tem sido muito útil para que as organizações atinjam um grau de maturidade no desenvolvimento de produtos e assim possam tentar equiparar-se as líderes de mercado em determinados sistemas tecnológicos. Como a modularidade decompõe e simplifica o complexo sistema de conhecimento, cada módulo tem a mesma base de conhecimento e está ligada a outros com a mesma interface, assim facilitando o processo de gestão da inovação nas organizações.

Como limitações desta pesquisa cita-se a utilização de apenas duas bases de dados para a pesquisa, que apesar da ampla utilização em trabalhos na área de engenharia, podem não cobrir todos os periódicos importantes de Sistemas de Inovação. Também não foi 


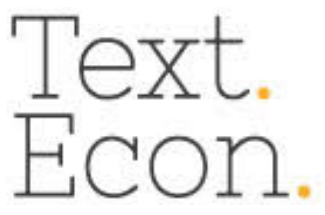

http://dx.doi.org/10.5007/2175-8085.2018v21n2p01

analisada a qualidade dos periódicos e das publicações selecionadas. A grande quantidade de artigos encontrados dificultou o processo de análise, contudo a busca com palavras-chaves mais restritas não foi eficaz, assim a análise pode ter sido conduzida de maneira errônea devido à discussão limitada de alguns tópicos. Além disso, a forma de análise e o enquadramento nos tipos de Sistemas de Inovação adotada cobre apenas a visão individual dos pesquisadores.

Nesse sentido, enfatiza-se a necessidade de maiores investigações tanto teóricas quanto empíricas para cada um dos níveis dos Sistemas de Inovação em particular, bem como a análise particular da modularidade dentro das funções dos Sistemas de Inovação. Estudos visando a identificação de políticas nacionais e regionais de incentivo a modularidade que podem beneficiar as organizações a serem mais eficazes e desenvolverem novos produtos beneficiando-se da sinergia das redes modulares podem ser bastante impactantes.

\section{REFERÊNCIAS BIBILIOGRÁFICAS}

ANDERSEN, P. H. Regional clusters in a global world: Production relocation, innovation, and industrial decline. California Management Review, v. 49, n. 1, p. 101-122, 2006.

BALDWIN, C. Y.; CLARK, K. B. Managing in an age of modularity. Harvard Business Review, v.75, p. 84-93, 1997.

BALDWIN, C.Y.; VON HIPPEL, E. Modeling a paradigm shift: From producer innovation to user and open collaborative innovation. Organization Science, v. 22, n. 6, p. 1399-1417, 2011.

BALDWIN, C. Y.; HENKEL, J. Modularity and intellectual property protection. Strategic Management Journal, v. 36, n. 11, p. 1637-1655, 2015.

BENASSI, M. Investigating modular organizations. Journal of Management \& Governance, v. 13, n. 3, p. 163-192, 2009.

BERGEK, A.; BERGGREN, C.; KITE Research Group. The impact of environmental policy instruments on innovation: A review of energy and automotive industry studies. Ecological Economics, v. 106, p. 112-123, 2014.

BOUNCKEN, R. B.; PESCH, R.; GUDERGAN, S. P. Strategic embeddedness of modularity in alliances: Innovation and performance implications. Journal of Business Research, v. 68, n. 7, p. 1388-1394, 2015.

CABIGIOSU, A.; CAMPAGNOLO, D.; FURLAN, A.; COSTA, G. Modularity in KIBS: The case of third-party logistics service providers. Industry and Innovation, v. 22, n. 2, p. 126-146, 2015. 
CABIGIOSU, A.; CAMUFFO, A. Beyond the "mirroring" hypothesis: Product modularity and interorganizational relations in the air conditioning industry. Organization Science, v. 23, n. 3, p. 686-703, 2012.

CABIGIOSU, A.; ZIRPOLI, F.; CAMUFFO, A. Modularity, interfaces definition and the integration of external sources of innovation in the automotive industry. Research Policy, v. 42, n. 3, p. 662-675, 2013.

CAMPAGNOLO, D.; CAMUFFO, A. The concept of modularity in management studies: a literature review. International Journal of Management Reviews, v. 12, n. 3, p. 259-283, 2010.

CHENG, L. V. Assessing performance of utilizing organizational modularity to manage supply chains: Evidence in the US manufacturing sector. International Journal of Production Economics, v. 131, n. 2, p. 736-746, 2011.

COOKE, P.; URANGA, M. G.; ETXEBARRIA, G. Regional innovation systems: Institutional and organisational dimensions. Research Policy, v. 26, n. 4, p. 475-491, 1997.

DANESE, P.; FILIPPINI, R. Direct and mediated effects of product modularity on development time and product performance. Engineering Management, IEEE Transactions on, v. 60, n. 2, p. 260-271, 2013.

DANESE, P.; FILIPPINI, R. Modularity and the impact on new product development time performance: Investigating the moderating effects of supplier involvement and interfunctional integration. International Journal of Operations \& Production Management, v. 30, n. 11, p. 1191-1209, 2010.

DAVIES, J.; JOGLEKAR, N. Supply chain integration, product modularity, and market valuation: Evidence from the solar energy industry. Production and Operations Management, v. 22, n. 6, p. 1494-1508, 2013.

DORAN, D.; HILL, A. A review of modular strategies and architecture within manufacturing operations. Proceedings of the Institution of Mechanical Engineers, Part D: Journal of Automobile Engineering, v. 223, n. 1, p. 65-75, 2009.

DORAN, D.; HILL, A..; HWANG, K.; JACOB, G. Supply chain modularisation: Cases from the French automobile industry. International Journal of Production Economics, v. 106, n. 1, p. 2-11, 2007.

EDQUIST, C. Systems of Innovation: perspectives and challenges. In: FAGERBERG, Jan; MOWERY, D. C.; NELSON, R. R. (Org). The Oxford Handbook of Innovation. Oxford/New York: Oxford University Press, 2004.

ETHIRAJ, S. K.; POSEN, H.E. Do product architectures affect innovation productivity in complex product ecosystems. Advances in Strategic Management, v. 30, p. 127-166, 2013.

FIXSON, S. K. Product architecture assessment: a tool to link product, process, and supply chain design decisions. Journal of Operations Management, v. 23, n. 3, p. 345-369, 2005.

FREEMAN, C. The 'National System of Innovation' in historical perspective. Cambridge Journal of Economics, v. 19, n. 1, p. 5-24, 1995.

FRIGANT, V.; LAYAN, J. Modular Production and the New Division of Labour Within Europe- The Perspective of French Automotive Parts Suppliers. European Urban and Regional Studies, v. 16, n. 1, p. 11-25, 2009. 


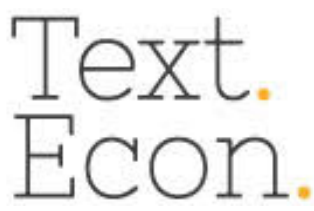

http://dx.doi.org/10.5007/2175-8085.2018v21n2p01

GODIN, B.. The Linear Model of Innovation: The historical construction of an analytical framework. Science Technology Human Values, v.31, p .639-667, 2006.

HARMANCIOGLU, N. Portfolio of controls in outsourcing relationships for global new product development. Industrial Marketing Management, v. 38, n. 4, p. 394-403, 2009.

HEKKERT, M.; NEGRO, S. Understanding Technological Change: Explanation of different perspectives on innovation and technological change. Utrecht University-Faculty of Geosciences, 2011.

HEKKERT, M.P.; SUURS, R.A.A.; NEGRO, S.O.; KUHLMANN, S.; SMITS, R. E. H. M. Functions of innovation systems: A new approach for analysing technological change. Technological Forecasting and Social Change, v. 74, p. 413-432, 2007.

HENKEL, J.; BALDWIN, C. Y.; SHIH, W. IP modularity: profiting from innovation by aligning product architecture with intellectual property. California Management Review, v.55, n. 4, p. 65-82, 2013.

HOETKER, G. Do modular products lead to modular organizations? Strategic Management Journal, v. 27, n. 6, p. 501-518, 2006.

JACOBS, M.; VICKERY, S. K.; DROGE, C. The effects of product modularity on competitive performance: do integration strategies mediate the relationship? International Journal of Operations \& Production Management, v. 27, n. 10, p. 1046-1068, 2007.

JACOBSSON, S.; JOHNSON, A. The diffusion of renewable energy technology: an analytical framework and key issues for research. Energy Policy, v. 28, n.9, p. 625-640, 2000.

JUROWETZKI, R.; LEMA, R.; LUNDVALL, B. Combining Innovation Systems and Global Value Chains for Development: Towards a Research Agenda. The European Journal of Development Research, p. 1-25, 2018.

KAMRAD, B.; SCHMIDT, G. M.; ÜLKÜ, S. Analyzing product architecture under technological change: Modular upgradeability tradeoffs. Engineering Management, IEEE Transactions on, v. 60, n. 2, p. 289-300, 2013.

KITCHENHAM, B. Procedures for Performing Systematic Reviews. Technical Report TR/SE-0401. Keele University, 2004.

LANGLOIS, R. N. Modularity in technology and organization. Journal of Economic Behavior \& Organization, v. 49, n. 1, p. 19-37, 2002.

LAU, A. K. W; YAM, R. C. M.; TANG, E. P.Y.; SUN, H.Y. Factors influencing the relationship between product modularity and supply chain integration. International Journal of Operations \& Production Management, v. 30, n. 9, p. 951-977, 2010.

LAU, A. K.W. Supplier and customer involvement on new product performance: contextual factors and an empirical test from manufacturer perspective. Industrial Management \& Data Systems, v. 111, n. 6, p. 910-942, 2011.

LIU, X.; XIE, Y.; WU, M. How latecomers innovate through technology modularization: Evidence from China's Shanzhai industry. Innovation, v. 17, n. 2, p. 266-280, 2015. 


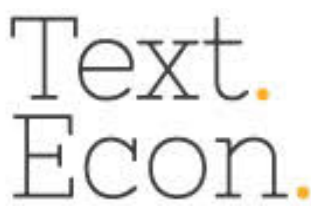

http://dx.doi.org/10.5007/2175-8085.2018v21n2p01

LUNDVALL, B.; JOHNSON, B.; ANDERSEN, E.S.; DALUM, Bent. National systems of production, innovation and competence building. Research Policy, v. 31, n. 2, p. 213-231, 2002.

MACCORMACK, A.; BALDWIN, C.; RUSNAK, J. Exploring the duality between product and organizational architectures: A test of the "mirroring" hypothesis. Research Policy, v. 41, n. 8, p. 1309-1324, 2012.

MALERBA, F. Sectoral systems of innovation and production. Research Policy, v. 31, n. 2, p. 247-264, 2002.

MALERBA, F. Sectoral systems: how and why innovation differs across sectors. In: FAGERBERG, J.; MOWERY, D. C.; NELSON, R. R. (Org). The Oxford Handbook of Innovation. Oxford/New York: Oxford University Press, 2004.

MCDERMOTT, G.; MUDAMBI, R.; PARENTE, R. Strategic modularity and the architecture of multinational firm. Global Strategy Journal, v. 3, n. 1, p. 1-7, 2013.

MENG, W. A Study of Textile Industry Supply Chain Based on Modularity Theory. Management \& Engineering, n. 20, p. 86, 2015.

MIKKOLA, J. H. Modularity, component outsourcing, and inter-firm learning. $R \& D$ Management, v. 33, n. 4, p. 439-454, 2003.

MLECNIK, E. Opportunities for supplier-led systemic innovation in highly energy-efficient housing. Journal of Cleaner Production, v. 56, p. 103-111, 2013.

NIOSI, J. Building national and regional innovation systems: institutions for economic development. United Kingdom: Edward Elgar Publishing, 2010.

OZMAN, M. Modularity, industry life cycle and open innovation. Journal of Technology Management \& Innovation, v. 6, n. 1, p. 26-34, 2011.

QI, Y.; WU, X.; CAO, J.; FAN, X. Flexible Knowledge Management on Modular Innovation Network. Advances in Information Sciences \& Service Sciences, v. 4, n. 19, 2012.

RAVISHANKAR, M. N.; PAN, S. L. Examining the influence of modularity and knowledge management $(\mathrm{KM})$ on dynamic capabilities: Insights from a call center. International Journal of Information Management, v. 33, n. 1, p. 147-159, 2013.

ROTABA, Z.; BEAUDRY, C. How Do High, Medium, And Low Tech Firms Innovate? A System of Innovation (SI) Approach. International Journal of Innovation and Technology Management, v. 9, n. 05, p. 1250034, 2012.

SALVADOR, F.; VILLENA, V. H. Supplier integration and NPD outcomes: Conditional moderation effects of modular design competence. Journal of Supply Chain Management, v. 49, n. 1, p. 87-113, 2013.

SHAMSUZZOHA, A. H. M.; KRISTIANTO, Y.; HELO, P. Implications of interface management for modularity degree. Journal of Modelling in Management, v. 8, n. 1, p. 6-24, 2013.

SANCHEZ, R.; MAHONEY, J. T. Modularity, flexibility, and knowledge management in product and organization design. Strategic Management Journal, v. 17, n. S2, p. 63-76, 1996. 
SCHILLING, M. A.; STEENSMA, H. K. The use of modular organizational forms: An industry-level analysis. Academy of Management Journal, v. 44, n. 6, p. 1149-1168, 2001.

SEURING, S.; MULLER, M.; WESTHAUS, M.; MORANA, R. Conducting a literature review - the example of sustainability in supply chains. In: KOTZAB, H.; SEURING, S.; MULLER, M.; REINER, G. (Eds.). Research Methodologies in Supply Chain Management. Physica, Heidelberg, p. 91-106, 2005.

SONG, W.; MING, X.; WANG, P. Collaborative product innovation network: Status review, framework, and technology solutions. Concurrent Engineering, v. 21, n. 1, p. 55-64, 2013.

STURGEON, T. J. Modular production networks: a new American model of industrial organization. Industrial and Corporate Change, v. 11, n. 3, p. 451-496, 2002.

SUN, Y.; GONG, X. Platformization Mechanism and Simulation Analysis of High-tech Service Innovation Network Based on Structural Holes Theory. International Journal of Security and its Applications, v. 9, n. 10, p. 209-220, 2015.

TAKEDA, Y.; KAJIKAWA, Y.; SAKATA, I.; MATSUSHIMA, K. An analysis of geographical agglomeration and modularized industrial networks in a regional cluster: A case study at Yamagata prefecture in Japan. Technovation, v. 28, n. 8, p. 531-539, 2008.

ÜLKÜ, S.; SCHMIDT, G.M. Matching product architecture and supply chain configuration. Production and Operations Management, v. 20, n. 1, p. 16-31, 2011.

ULRICH, K. The role of product architecture in the manufacturing firm. Research Policy, v. 24, n. 3, p. 419-440, 1995.

VIND, I.; FOLD, N. Multi-level modularity vs. hierarchy: global production networks in Singapore's electronics industry. Geografisk Tidsskrift-Danish Journal of Geography, v. 107, n. 1, p. 69-83, 2007.

ZESCHKY, M.; DAIBER, M.; WIDENMAYER, B.; GASSMANNA O. Coordination in global R\&D organizations: an examination of the role of subsidiary mandate and modular product architectures in dispersed R\&D organizations. Technovation, v. 34, n. 10, p. 594-604, 2014.

ZHANG, G.; GAO, R. Modularity and incremental innovation: the roles of design rules and organizational communication. Computational and Mathematical Organization Theory, v. 16, n. 2, p. 171-200, 2010.

ZHU, F.; XIU-XIA, S.; JANIS, M.; ZHI-JUN, D. Innovations in Knowledge Management: Applying Modular Design. International Journal of Innovation Science, v. 6, n. 2, p. 83-96, 2014.

ZIRPOLI, F.; BECKER, M. C. The limits of design and engineering outsourcing: performance integration and the unfulfilled promises of modularity. $R \& D$ Management, v. 41, n. 1, p. 21-43, 2011. 